\title{
Contralateral Eyebrow Approach for Unilateral Suprasellar Meningioma
}

\author{
Sorin Aldea ${ }^{1}$ Stéphan Gaillard ${ }^{1}$ \\ ${ }^{1}$ Department of Neurosurgery, Foch Hospital, Suresnes, France \\ J Neurol Surg B 2018;79(suppl S2):S215-S217.
}

\begin{abstract}
Address for correspondence Sorin Aldea, MD, Department of Neurosurgery, Foch Hospital, 40 rue Worth, 92150 Suresnes, France (e-mail: s.aldea@hopital-foch.org).
\end{abstract}

\begin{abstract}
Keywords

- suprasellar tumor

- eyebrow approach

- contralateral approach

- meningioma

Unilateral suprasellar meningiomas have distinct features compared to other midline tumors, as they may produce severe visual symptoms even if small due to an early involvement of the optic canal. Surgical treatment of these tumors from an ipsilateral approach is challenging, as the tumor is covered by the optic nerve that needs to be mobilized to access the optic canal extension. A contralateral approach allows a direct line of sight to the tumor despite a longer working distance. We report the case of a 49year-old patient presenting with unilateral visual loss related to a left suprasellar meningioma extending to the left optic canal and displacing the optic nerve laterally. Through a right eyebrow approach, a $2.5 / 2 \mathrm{~cm}$ supraorbital bone flap was raised and the orbital floor was thoroughly flattened. After dural opening, the carotid cistern was opened and CSF evacuated allowing a surgery without fixed retractors. The intracranial part of the tumor was removed, but the optic nerve seemed to be still displaced by the intracanalicular part. Under copious irrigation, the medial part of the optic canal was drilled, the dura incised, and the tumor removed. Postoperative course was favorable and the patient made a complete visual recovery. Postoperative MRI showed complete removal of the tumor. We present different surgical steps and discuss the nuances of the procedure. The contralateral eyebrow approach is an interesting addition to the surgical armamentarium and should be discussed for unilateral suprasellar tumors. The link to the video can be found at: https://youtu.be/2LTEOaGoKzo.
\end{abstract}

Conflict of Interest

None.

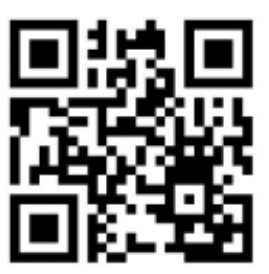

www.thieme.com/skullbasevideos

www.thieme.com/jnlsbvideos received

October 12, 2017

accepted

December 7, 2017

published online

January 19, 2018
DOI https://doi.org/

10.1055/s-0038-1623520.

ISSN 2193-6331.
๑) 2018 Georg Thieme Verlag KG
Stuttgart · New York

License terms

(c) (1) $\ominus$ (5) 


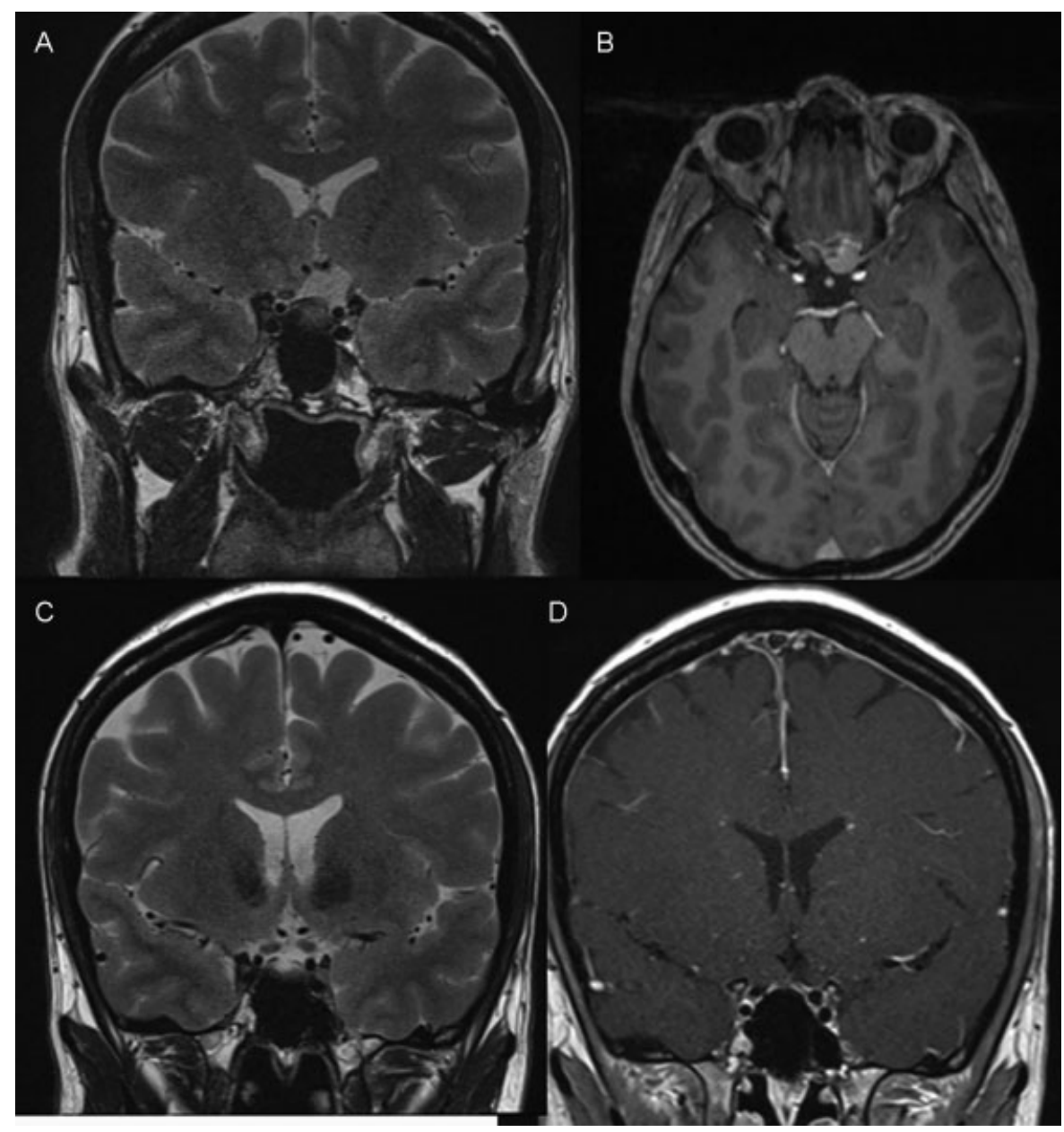

Fig. 1 (A) Preoperative T2 weighted coronal MRI showing a hyper intense left suprasellar meningioma compressing and displacing laterally the left optic nerve. (B) Preoperative axial T1 weighted gadolinium enhanced MRI showing extension of the tumor in the left optic canal. (C) and (D) Postoperative coronal T2 weighted and axial T1 weighted gadolinium enhanced MRI show complete removal of the tumor and decompression of the left optic nerve. 


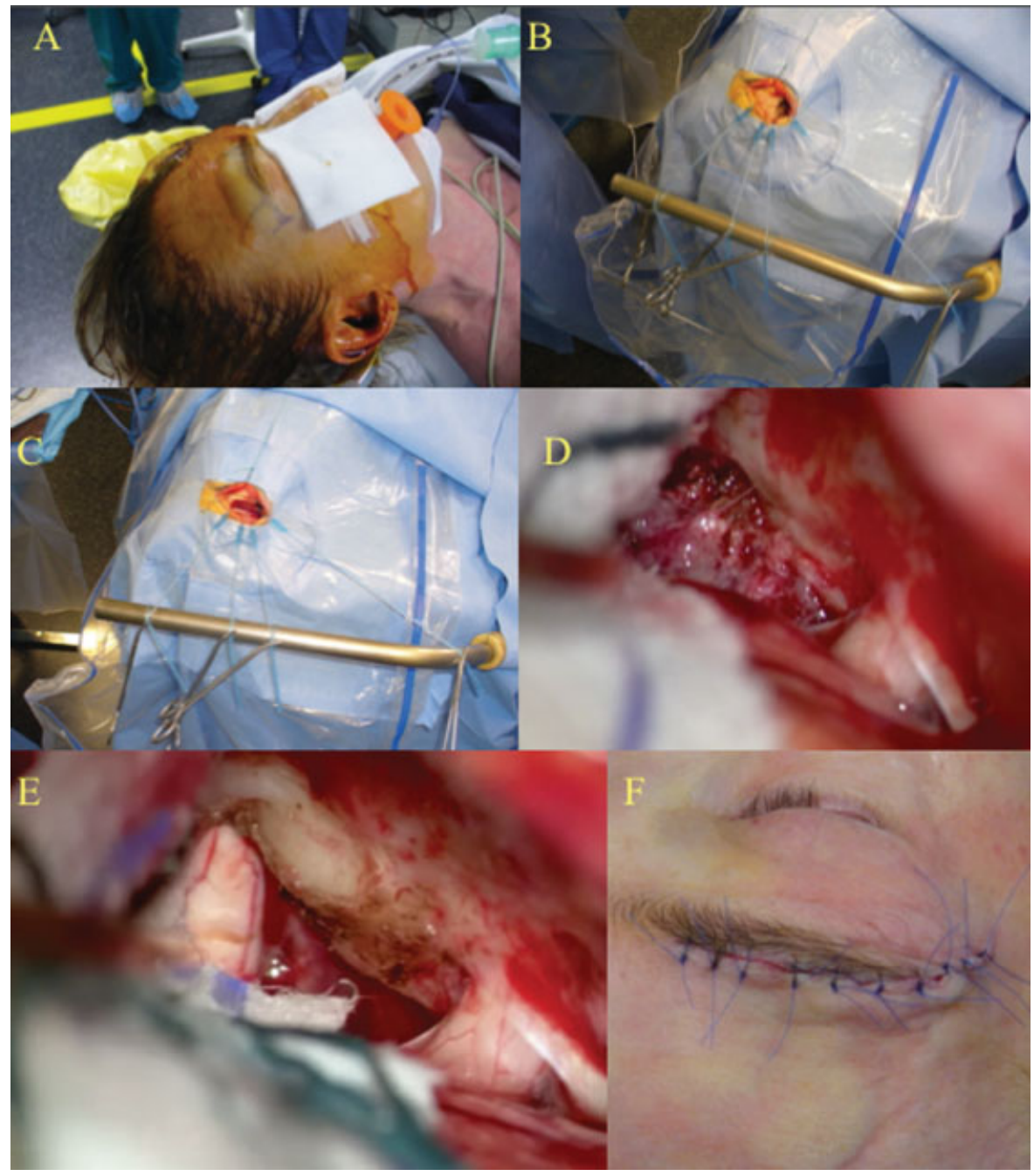

Fig. 2 Illustrative surgical steps of the right contralateral eyebrow approach for removal of a left suprasellar meningioma. (A) Positioning (B) Skin exposure (C) Supraorbital bone flap (D) Initial exposure and decompression of the meningioma (E) Final result after removal of the tumor and opening of the contralateral optic canal (F) Skin closure. 